\title{
Treinamento e desenvolvimento de pessoal de enfermagem: um modelo de planilha de custos*
}

\author{
TRAINING AND DEVELOPMENT OF THE NURSING STAFF: A MODEL OF SPREAD SHEET COST \\ ENTRENAMIENTO Y ACTUALIZACIÓN DEL PERSONAL DE ENFERMERÍA: \\ UN MODELO DE PLANILLA DE COSTOS
}

\section{Marli de Carvalho Jerico', Valéria Castilho²}
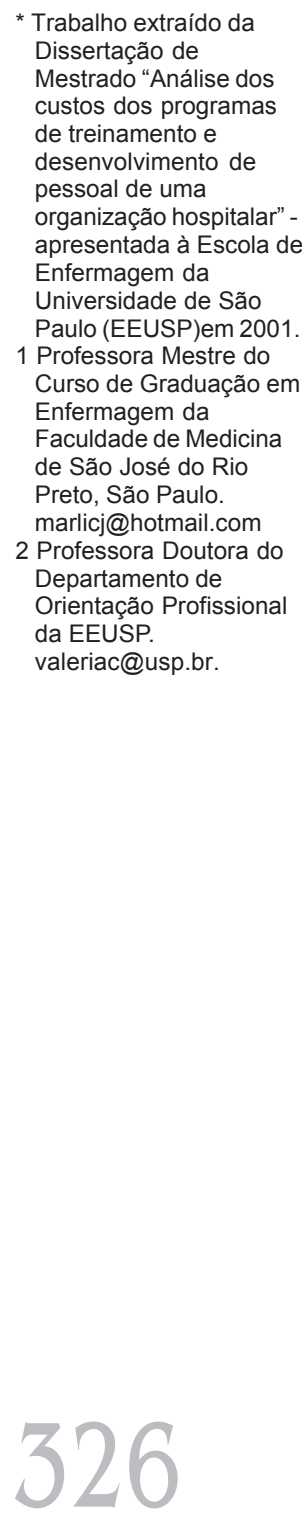

Rev Esc Enferm USP 2004; 38(3): 326-31.

\author{
RESUMO \\ Este artigo apresenta um \\ modelo de planilha de \\ composição de custos para \\ programas de treinamento e \\ desenvolvimento de pessoal \\ em uma organização \\ hospitalar. Para sua \\ elaboração foram \\ considerados os elementos \\ relevantes na composição do \\ custo total dos programas de \\ treinamento. A planilha \\ proposta está dividida em \\ quatro partes: \\ Item 1 - dados relativos aos \\ programas de treinamento; \\ Item 2 - custos diretos desses \\ programas; \\ Item 3 - custos indiretos \\ (estrutura do CEC) $e$ \\ Item 4 - total dos custos. \\ A utilização de planilhas de \\ custos auxiliam no \\ conhecimento e gerenciamento \\ dos mesmos por parte dos \\ enfermeiros, gerentes de \\ ABSTRACT \\ This paper presents a model \\ of spread sheet cost for \\ training and development \\ programs to the nursing \\ staff in a hospital \\ organization. Significant items \\ of the total cost have been \\ considered in relation to its \\ elaboration and proper \\ functioning. This model is \\ divided into four parts: \\ Item 1 - data related to the \\ training programs; \\ Item 2 -direct costs of these \\ programs; \\ Item 3 -indirect costs \\ (Continuum Educational \\ Center structure), and \\ Item 4 - cost amount. \\ The use of spread sheet cost \\ may provide knowledge and \\ management for the nurses \\ and nurse managers. \\ However, the related costs \\ should be reviewed \\ according to each service.
} unidades. No entanto, para sua aplicação, devem ser revistos os custos que serão agregados, conforme especificidade de cada serviço.

\section{PALAVRAS-CHAVE}

Custos e análise de custo. Instituições de saúde. Desenvolvimento de pessoal. Capacitação em serviço.

\section{KEYWORDS}

Costs and cost analysis.

Health facilities.

Staff development.

Training inservice.

\section{RESUMEN}

Este artículo expone un modelo de planilla de composición de costos para programas de entrenamiento y actualización en una organización hospitalaria. Para su elaboración se consideraron los elementos relevantes en la composición del costo total de los programas de entrenamiento. La planilla propuesta está dividida en cuatro partes: Item 1 -dados relativos a los programas de entrenamiento; Item 2 - costos directos de esos programas; Item 3-costos indirectos (estructura del CEC) $y$ Item 4 -costos totales. La utilización de planillas de costos contribuye al conocimiento e gerenciamiento de los mismos por parte de los enfermeros, gerentes de unidades. En tanto, para su aplicación, deben ser revisados los costos que serán añadidos, conforme a la especificidad de cada servicio.

\section{PALABRAS CLAVE}

Costos y análisis de costo. Instituciones de salud. Desarrollo de personal. Capacitación en servicio. 


\section{INTRODUÇÃO}

Atualmente, muitos enfermeiros, no cotidiano das suas atividades gerenciais, estão começando a se preocupar em dispor de informações sobre custos. Assim, nas negociações com a administração geral, podem respaldar seus argumentos em relação à necessidade de investimentos em treinamento, bem como justificar suas decisões sobre a alocação de recursos com tal propósito.

De outro lado, estudar os custos dos programas de Treinamento e Desenvolvimento (T \& D), representa também preocupação com a qualidade da assistência prestada aos usuários da instituição e com a qualificação e capacitação dos funcionários que nela atuam.

A propósito, em 1993, o Conselho Internacional de Enfermagem, afirmou que as finanças constituem outro domínio de conhecimento da enfermagem, recomendando aos profissionais que promovam investigações para validar metodologias de cálculo de custos ${ }^{(1)}$.

A informação de custos como insumo fundamental do gerenciamento de enfermagem, constitue-se no destaque do movimento gerencial na atualidade. Deve ser compreendida não só como uma questão técnica ou econômica, mas uma questão comportamental, pois representa o desenvolvimento de uma gestão competente e compatível com a missão de cuidar da saúde da população( ${ }^{(2)}$.

A compreensão do comportamento dos custos é extremamente interessante à avaliação do desempenho, à tomada de decisões e ao planejamento das atividades operacionais. Assim, é possível avaliar a viabilidade dos serviços, as alternativas de investimentos e a repercussão de modificações no volume de atendimentos ${ }^{(3)}$.

Diante disto, é necessário lembrar a importância do custo como ferramenta gerencial para o enfermeiro do Centro de Educação Continuada (CEC), pois atende as finalidades de controle, fornece suporte para a análise de viabilidade econômica de um projeto e ampara o processo decisório em relação a alocação de recursos e à redução de custos, sem prejuízo da qualidade.

É possível observar que várias instituições de saúde não possuem uma política de investimentos formalizada, e nem os CECs possuem previsão orçamentária voltada para a manutenção de um programa de educação continuada.

No entanto, é de responsabilidade da gerência de treinamento, predeterminar as seqüências de sua ação administrativa com vistas à definição orçamentária da unidade de treinamento, com a respectiva fixação de recursos financeiros para os programas de desenvolvimento de recursos humanos ${ }^{(4)}$.

Uma das dificuldades referidas pelos enfermeiros, na análise dos custos de programas de T\&D, é definir todos os gastos que estão vinculados a tal programação. Também, não dispõem de um roteiro sistematizado, levando a registros precários ou anotações esparsas de alguns itens dentro de uma programação.

Devido a esses aspectos torna-se necessária a elaboração de uma planilha de custos que contenha dados relevantes às necessidades do trabalho diário do enfermeiro. Além disso, esses dados podem ser utilizados na avaliação de desempenho gerencial da unidade.

Elaborar e propor uma planilha de composição de custos para programas de treinamentos, que sirva como referência para enfermeiros nesta área, principalmente, para enfermeiros de centros de educação continuada, constituiu uma parte da dissertação de mestrado sobre custos dos programas de treinamentos desenvolvidos em um CEC de uma instituição hospitalar do interior do Estado de São Paulo ${ }^{(5)}$. Assim, nosso objetivo é divulgar a elaboração e a proposta da referida planilha.

\section{DESENVOLVIMENTO}

Para a elaboração da planilha levou-se em conta os elementos relevantes na composição do custo total dos programas de treinamento.

No estudo em questão, configuramos custo direto aqueles gastos consumidos diretamente na execução do treinamento, ou seja, com instrutores, materiais, recursos audiovisuais e despesas gerais do treinamento, não havendo necessidade de rateio.

Já o custo indireto, não se refere ao treinamento especificamente e sim às condições necessárias para a execução do treinamento, representado pela estrutura (energia elétrica, custo administrativo em geral, depreciação e outros) do CEC.
Treinamento e desenvolvimento

de pessoal de enfermagem: um modelo de planilha de custos 
Marli de Carvalho Jerico Valéria Castilho
A planilha proposta está dividida em quatro itens que contém os seguintes dados: relativos aos programas de treinamento (item 1); aos custos diretos desses programas (item 2); aos custos indiretos, ou seja, da estrutura do CEC (item 3 ) e o total dos custos (item 4) (ver ANEXO).

O item 1 é composto de dados do programa, com o nome do programa, período, carga horária, horário, objetivos do treinamento, total de participantes, número de ausentes, categoria funcional (total de funcionários e função), salário categoria/mês em reais $(R \$)$, benefícios ( $\mathrm{R} \$)$, horas de trabalho por mês, encargos sociais por hora de trabalho, salário da categoria por hora de trabalho, custo total da categoria por hora de trabalho e custo total dos participantes.

Estes dados foram importantes para aferir, principalmente, o investimento indireto, considerado como horas disponibilizadas de recursos humanos para a aprendizagem.

O item 2 da planilha consta de dados dos custos diretos, os quais estão subdivididos em custos com: 2.1 - instrutor, 2.2 - material de consumo, 2.3 - equipamento e locação e 2.4 - despesas gerais.

Em relação aos custos do subitem 2.1- instrutor - assinalar se este é interno ou externo, se remunerado ou não. Também, deve anotar: as despesas com alimentação, transporte, pedágio, estacionamento, certificado e outros; a remuneração hora e total do instrutor, bem como o custo do instrutor (remuneração mais despesas).
No que concerne ao subitem 2.2 - material de consumo ou insumos - são registrados os materiais utilizados nos programas como: apostila, xerox, pastas, canetas, transparência, marketing e folhetos, slides, fita de vídeo, papel para flip-chart, pincel atômico, giz, certificado e outros materiais específicos para determinados treinamentos.

O subitem 2.3 - equipamento e locação compreende os recursos audiovisuais (rav) elencados como projetor de slides, televisão (tv), vídeo, retroprojetor e outros.

O subitem 4 - despesas gerais - que neste caso consiste em despesas com alimentação, como aquelas agrupadas como "coffee-break" (padronizadas pelo Serviço de Nutrição e Dietética do hospital), café e copos descartáveis.

Ao final de cada um dos subitens orienta-se registrar o custo unitário, a quantidade e o custo total de cada um, seguido do custo total. Assim, a soma do custo total dos itens perfaz o total dos custos diretos utilizados em um treinamento.

Constitui o item 3 da planilha, os custos indiretos, ou seja, os custos da estrutura do CEC nos quais foram digitados o custo do rateio por hora, as horas de treinamento e o custo total de cada um, seguido do custo total dos custos indiretos. No ítem 4 da planilha são registrados o total dos custos, ou seja, custos diretos e custos indiretos, o custo total e custo hora de cada um, resultando no total geral dos custos e o custo total/hora.

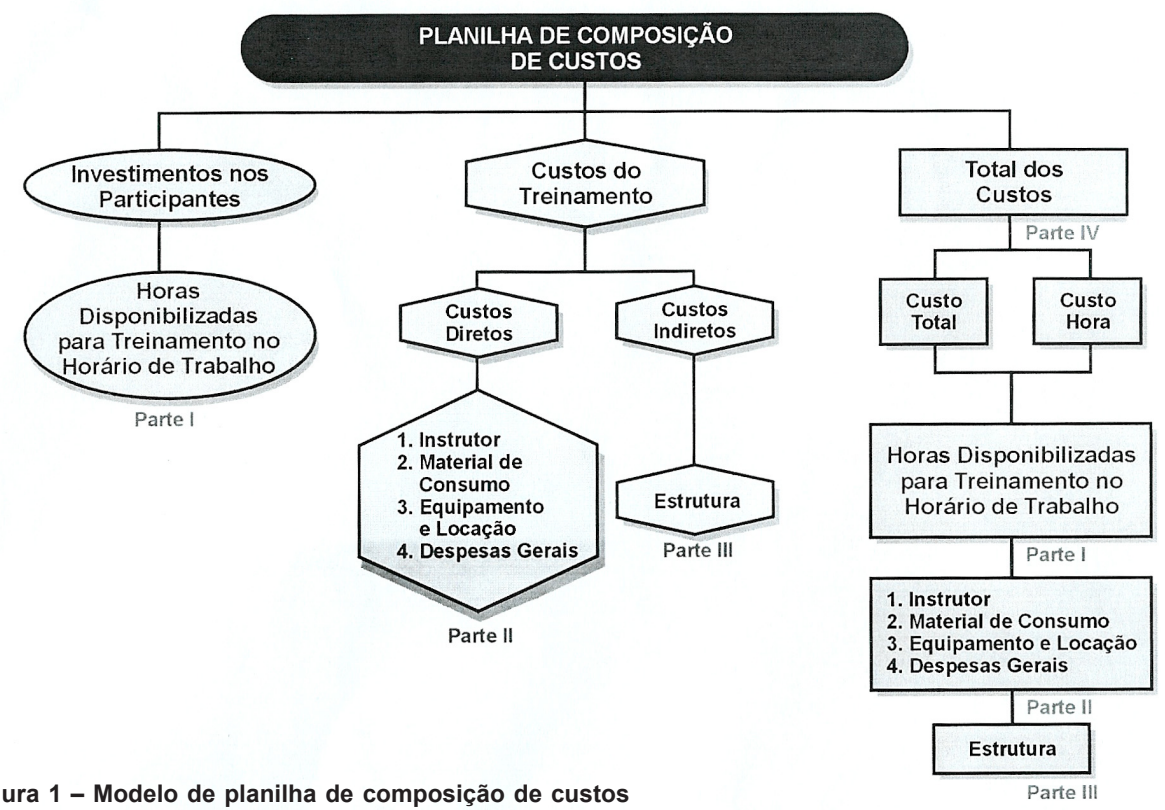




\section{CONSIDERAÇÕES FINAIS ERECOMENDAÇÕES}

Sugere-se que os dados sejam processados eletronicamente, por exemplo, no programa Microsoft Access 2000, pois gera informações que atendem aos propósitos gerenciais de um CEC.

Também, para a análise de dados pode-se utilizar o software Microsoft Excel versão 2000 para o ambiente Windows que dispõe das variáveis numéricas: somatória, média aritmética, mediana, percentual e outros.

A primorar a prática da enfermagem é questão que tem merecido muitas discussões e reflexões no nosso meio profissional, bem como participação, competência e qualificação dos enfermeiros em gerenciar informações de ordem econômico-financeira de suas atividades diárias, com vistas a encontrar caminhos que fortaleçam a função gerencial na apresentação de resultados.
A elaboração de planilhas como instrumento para a análise de custos, sem dúvida auxilia na produção de relatórios financeiros, propiciando análise do ciclo operacional dos treinamentos, como também o controle dos custos.

O uso da tecnologia para desenvolver planilhas de custo, proporciona a transformação dos dados em informações que auxiliarão os gerentes dos CECs a otimizarem os recursos disponíveis e a maximizarem a produtividade, preservando a qualidade das atividades desenvolvidas.

Pesquisas futuras serão necessárias para desenvolver mais o conhecimento do enfermeiro na área econômica. Esperamos também que a planilha de custos proposta seja aperfeiçoada, através de sua aplicação em outras instituições, cujos custos se conformarão de acordo com as suas especificidades.

\section{REFERÊNCIAS}

(1) Consejo Internacional de Enfermeras. La calidad los costos y la enfermería. Ginebra: CIE; 1993. [Carpeta del Dia Internacional de la Enfermera]

(2) Matos AJ. Repensando o custeio do serviço de enfermagem. São Paulo: Planisa; 1995.

(3) Lima CRM. Activity based costing para Hospitais. [dissertação] São Paulo (SP): Escola de Administração de Empresas de São Paulo/ FGV; 1997.
(4) Carvalho AV. Administração de recursos humanos. 2a ed. São Paulo: Pioneira; 1997.

(5) Jericó MC. Análise dos custos dos programas de treinamento e desenvolvimento de pessoal de uma organização hospitalar. [dissertação] São Paulo (SP): Escola de Enfermagem da USP; 2001.
Treinamento e desenvolvimento de pessoal de enfermagem: um modelo de planilha de custos 
Marli de Carvalho Jerico Valéria Castilho
ANEXO

PLANILHA DE COMPOSIÇÃO DOS CUSTOS DE TREINAMENTO

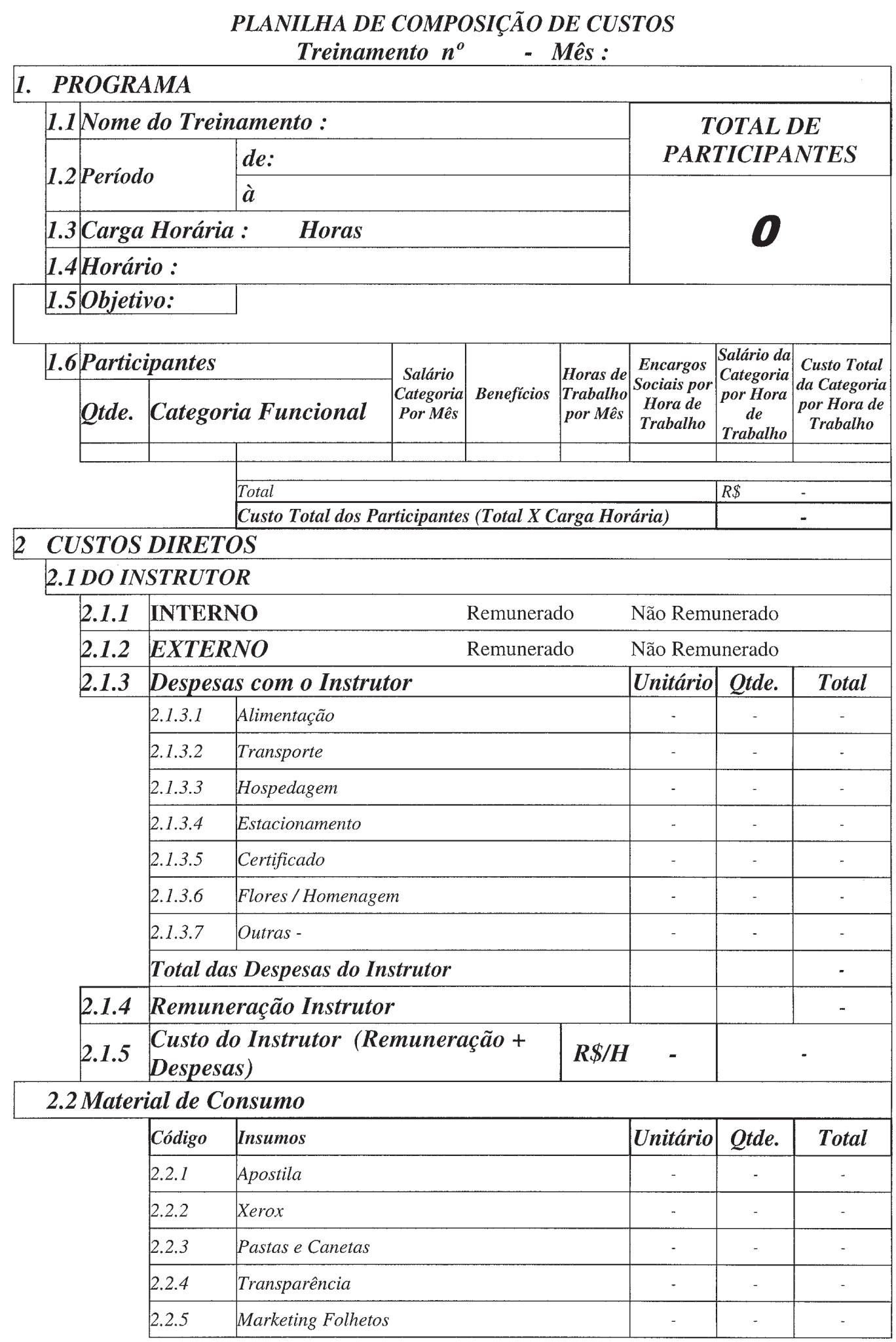




\subsection{Material de Consumo}

\begin{tabular}{|l|l|c|c|c|}
\hline 2.2 .6 & Serviços Gráficos & - & - & - \\
\hline 2.2 .7 & Slides & - & - & - \\
\hline 2.2 .8 & Fita de vídeo & - & - & - \\
\hline 2.2 .9 & Papel para Flip Chart & - & - & - \\
\hline 2.2 .10 & Giz & - & - & - \\
\hline 2.2 .11 & Certificado & - & - & - \\
\hline 2.2 .12 & Flip Chart & - & - & - \\
\hline 2.2 .13 & Pincel atômico & - & - & - \\
\hline 2.2 .14 & Outros - & - & - & - \\
\hline
\end{tabular}

\subsection{Equipamento e Locação}

\begin{tabular}{|l|l|c|c|c|}
\hline Código & Insumos & Unitário & Qtde. & Total \\
\hline 2.3 .1 & Projetor de Slides & - & - & - \\
\hline 2.3 .2 & Tv/Vídeo & - & - & - \\
\hline 2.3 .3 & Retroprojetor & - & - & - \\
\hline 2.3 .4 & Computador & - & - & - \\
\hline 2.3 .5 & Canhão de Projeção & - & - & - \\
\hline 2.3 .6 & Outros & - & - & - \\
\hline & Total dos Recursos Audiovisuais & & & - \\
\hline
\end{tabular}

\subsection{Despesas Gerais}

\begin{tabular}{|l|l|c|c|c|}
\hline Código & Insumos & Unitário & Qtde. & Total \\
\hline 2.4 .1 & Coffee-Break & - & - & - \\
\hline 2.4 .2 & Café & - & - & - \\
\hline 2.4 .3 & Copos descartáveis & - & - & - \\
\hline & Total das Despesas Gerais & \multicolumn{2}{|c}{-} \\
\hline
\end{tabular}

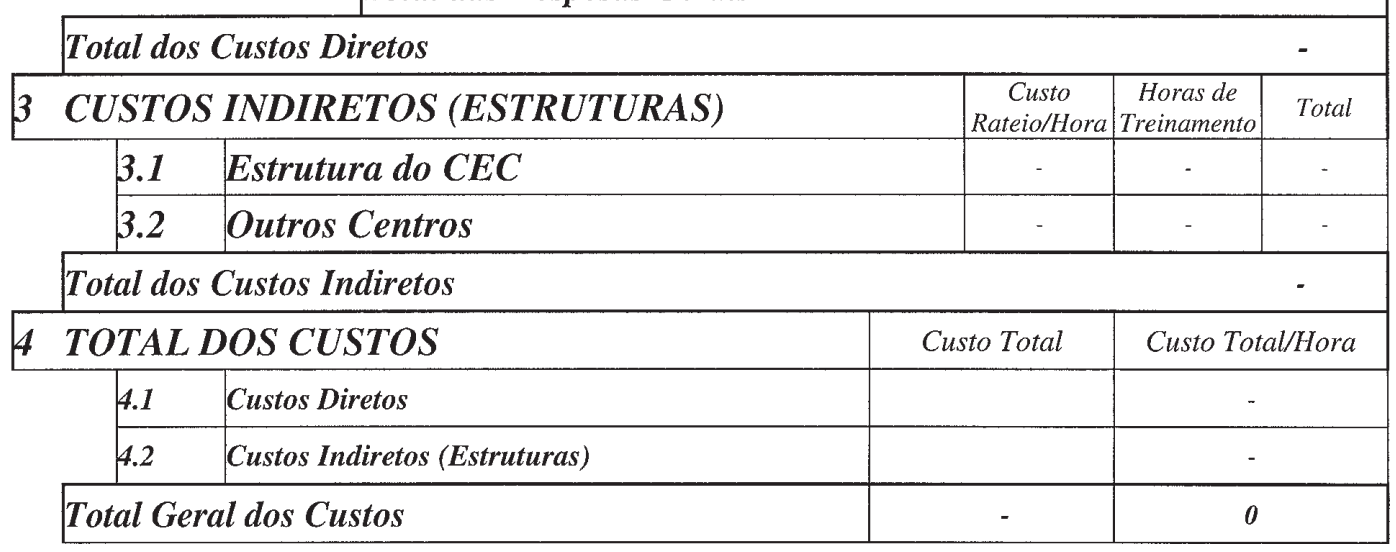

